\title{
Questionário de Procrastinação Acadêmica - Consequências negativas: propriedades psicométricas e evidências de validade
}

\author{
Gabriela Ballardin Geara, Marco Antônio Pereira Teixeira ${ }^{1}$ \\ Universidade Federal do Rio Grande do Sul - Porto Alegre-RS, Brasil
}

\section{RESUMO}

A procrastinação acadêmica é uma forma de adiamento da realização de tarefas acadêmicas, considerada uma falha no processo de autorregulação dos estudantes. O objetivo deste estudo foi construir um instrumento para avaliar a percepção de universitários sobre diversos aspectos da procrastinação acadêmica: impacto geral na vida, consequências negativas (acadêmicas, físicas e psicológicas), motivação para reduzi-la e para receber ajuda com isso. Também procurou-se obter evidências de validade do instrumento por meio de análise de componentes principais e de correlações com desempenho acadêmico e autorregulação da aprendizagem dos universitários. Participaram do estudo 534 universitários que responderam ao instrumento via internet. Análises de componentes principais e correlações com autorregulação da aprendizagem e desempenho acadêmico forneceram boas evidências de validade e de fidedignidade para o instrumento. Conclui-se que o instrumento pode ser usado em pesquisas e intervenções voltadas ao público universitário.

Palavras-chave: validade do teste; estudantes universitários; construção do teste.

\section{ABSTRACT - Academic Procrastination Questionnaire - Negative consequences: Psychometric properties and evidence for validity}

The academic procrastination is a way of postponing the fulfillment of academic tasks, considered a failure in the self-regulation process. This study aimed to construct an instrument to evaluate the perception of the college students about their academic procrastination regarding the overall impact of it, its frequency of occurrence, the frequency of its negative consequences (academic, physical and psychological) and their motivation to reduce it and to receive help. Validity evidences were also explored through principal component analysis and correlations with academic performance and self-regulation. The participants were 534 college students who answered the questionnaire by the internet. Good evidences of validity of the instrument were given by principal component analysis and correlation tests with self-regulation of learning and academic performance. The reliability of its subscales assessed through internal consistency also proved to be adequate. It is concluded that the instrument can be useful in research and in interventions with college students.

Keywords: test validity; college students; test construction.

RESUMEN - Cuestionario de Procrastinación Académica - Consecuencias negativas: propiedades psicométricas y evidencias de validez

La procrastinación académica es una manera de postergar la realización de tareas académicas — entendida como una falla en el proceso de autorregulación de los estudiantes. El objetivo de este estudio fue construir un instrumento para evaluar la percepción de los estudiantes sobre diversos aspectos de la procrastinación académica: impacto general sobre la vida, sus consecuencias negativas (académicas, físicas y psicológicas) y la motivación para reducirla y para recibir ayuda. Fueron exploradas evidencias de validez del instrumento a través del análisis de componentes principales y correlación con rendimiento académico y autorregulación del aprendizaje de los universitarios. Los participantes del estudio fueron 534 estudiantes universitarios que respondieron al instrumento en internet. Análisis de componentes principales y las correlaciones con autoregulación del aprendizaje y desempeño académico proporcionaron buenas evidencias de validez y de fidegnidad para el instrumento. Se concluye que el instrumento se puede utilizar en investigaciones e intervenciones dirigidas a los estudiantes universitarios.

Palabras clave: validación de test; estudiantes universitarios; construcción de test. 
De modo geral, a procrastinação pode ser entendida como o adiamento voluntário de uma atividade pretendida, necessária e/ou com importância pessoal, apesar de o sujeito esperar potenciais consequências negativas que superariam as consequências positivas desse adiamento (Klingsieck, 2013). Essa definição foi elaborada por Klingsieck (2013) a partir de uma extensa revisão de literatura na qual estava inclusa a meta-análise de 216 trabalhos conduzida por Steel (2007). O comportamento de procrastinar as mais diversas tarefas da vida em geral tem se correlacionado com ansiedade, depressão, impulsividade, perfeccionismo, conscienciosidade, aversividade da tarefa, baixo desempenho acadêmico, baixa autoestima, baixa autoeficácia, baixo autocontrole, entre outros (Steel, 2007).

A procrastinação tem sido considerada uma falha nos processos de autorregulação do comportamento (Steel \& Klingsieck, 2016). A autorregulação é o processo consciente e voluntário por meio do qual o indivíduo faz a gestão dos seus próprios comportamentos, pensamentos e sentimentos de um modo cíclico e direcionado à realização de metas pessoais (Bandura, 1991). Apesar dessa capacidade de autorregulação ser necessária em diversos aspectos da vida, pesquisas mostram que as pessoas têm apresentado problemas decorrentes de falhas nesse processo como, por exemplo, excessos no consumo de alimentos, de produtos e/ou serviços (Steel, 2007). Entres as falhas comuns de autorregulação, encontra-se a procrastinação de diversos tipos de tarefas, cujo ponto central do fenômeno está na dificuldade de conectar intenção e ação (Steel, 2007). Isso implica dizer que o indivíduo que procrastina tem dificuldade de implementar aquilo que deseja ou planeja (Steel, 2007).

$\mathrm{Na}$ maioria dos estudos, é evidente o destaque ao desconforto emocional e/ou às psicopatologias possivelmente relacionadas à procrastinação acadêmica (Steel, 2007). Quem procrastina considera esse seu comportamento como algo ruim, prejudicial e tolo (Steel, 2007), sendo que a maioria dos indivíduos manifesta o desejo de mudar esse comportamento (Solomon \& Rothblum, 1984). No entanto, essa modificação comportamental pode não ser algo simples, já que tal comportamento é afetado por aspectos pessoais, comportamentais e ambientais, dependendo da interação entre o indivíduo, a tarefa e o contexto em que está inserido ao longo de sua vida (Schouwenburg, 2004).

Os primeiros estudos sobre as consequências da procrastinação surgiram na década de 1980 (Steel, 2007). No entanto, o conhecimento acumulado sobre esse tema ainda é fragmentado, e somente mais recentemente houve um aumento do número de estudos publicados sobre as consequências negativas da procrastinação (Steel \& Klingsieck, 2016; Rebetez, Barsics, Rochat, D’Argembeau, \& Linden, 2016). A maioria dos estudos possui caráter exploratório, incluindo metodologias quantitativas e qualitativas (Grunschel, Patrzek, \& Fries, 2013; Hussain \& Sultan, 2010; Steel \& Klingsieck, 2016).
Um dos contextos nos quais a procrastinação e suas consequências têm sido estudadas é o acadêmico, especialmente o do ensino superior, caracterizando o que é chamado de procrastinação acadêmica. No ensino superior, em contraste aos níveis de ensino mais básicos, existem mais tarefas a serem realizadas em um período de tempo limitado (Couto, 2014). Isso faz com que sejam exigidas mais autonomia e autorregulação dos estudantes em relação ao seu processo de aprendizagem (Polydoro et al., 2015).

Apesar disso, a procrastinação acadêmica é um comportamento comum: estudos sugerem que de 70 (Ferrari, O'Callaghan \& Newbegin, 2005) a 95\% (O’Brien, 2002) dos estudantes do ensino superior procrastinam. Além disso, a procrastinação pode ocorrer de forma prolongada e sistemática — o que pode estar relacionado às evidências de que entre 20 (Schouwenburg, 1995) e $50 \%$ dos universitários percebem que procrastinam suas tarefas acadêmicas de forma problemática (Solomon \& Rothblum, 1984).

Em relação à procrastinação acadêmica, não há consenso entre os pesquisadores quanto a uma possível categorização de suas consequências negativas. No entanto, a maior parte dos estudos aprofunda a avaliação das consequências nos aspectos acadêmico, físico e psicológico da vida dos universitários (Grunschel et al., 2013; Hussain \& Sultan, 2010; Krause \& Freund, 2014).

A procrastinação acadêmica ainda é um tema pouco explorado no Brasil. Em 10 de fevereiro de 2016, em um levantamento realizado pelos autores deste trabalho com o descritor "procrastinação" nas bases de dados SciELO, LILACS, Biblioteca Virtual de Saúde, PePSIC, IndexPSI, MedLine, Banco de Teses da CAPES e Portal de Periódicos da CAPES, considerando todo o período disponível nas bases, foram localizados 15 diferentes estudos publicados em Português. Tais estudos abordavam a procrastinação em diferentes contextos, a partir de perspectivas de áreas tais como Psicologia, Educação, Administração e Economia. Por outro lado, no PsycInfo, uma das bases de dados mais abrangentes da psicologia mundial, a busca feita com o descritor "procrastination" gerou 937 resultados para todo o período disponível para a pesquisa - incluindo diversos instrumentos publicados sobre o tema em Inglês, tais como Procrastination Assessment Scale for Students (Solomon \& Rothblum, 1984), Tuckman Procrastination Scale (Tuckman, 1991) e Pure Procrastination Scale (Steel, 2010).

No Brasil, no que se refere à avaliação da procrastinação em geral (ou seja, sem especificar um tipo de tarefa ou contexto), localizou-se apenas a Escala de Procrastinação Ativa (Gouveia, Pessoa, Coutinho, Barros, \& Fonseca, 2014), que avalia características do comportamento de procrastinar, tais como decisão intencional, preferência por pressão, satisfação com os resultados e habilidade em cumprir prazos. Para avaliar a procrastinação de tarefas de estudo, localizou-se somente a Escala de 
Procrastinação Acadêmica (Sampaio, 2011), que avalia a procrastinação do estudo para provas e do estudo diário. Entretanto, esses instrumentos não avaliam a percepção do indivíduo sobre o desconforto que ele experimenta por agir dessa forma nem as possíveis consequências negativas do seu comportamento procrastinatório - o que pode evidenciar uma lacuna para o desenvolvimento de pesquisas sobre esse tema.

O objetivo desta pesquisa foi, então, construir um instrumento para avaliar a percepção de estudantes universitários sobre possíveis consequências negativas da procrastinação acadêmica. Perceber os efeitos negativos de um comportamento é o primeiro passo para que ele possa ser modificado. Assim, o instrumento proposto visa não somente mensurar as consequências negativas da procrastinação, mas também propiciar a quem o responde uma reflexão sobre o seu comportamento procrastinatório. Para isto, propôs-se a criação de um instrumento que avaliasse:

- $\quad$ as tarefas acadêmicas mais procrastinadas;

- o nível de impacto geral da procrastinação acadêmica na vida dos universitários;

- o grau de possíveis consequências negativas da procrastinação acadêmica nos âmbitos acadêmico, físico e psicológico; e

- o nível de motivação para mudar o comportamento procrastinatório (em termos da motivação para reduzir a frequência dos comportamentos relacionados à procrastinação acadêmica e para receber ajuda com relação a isso).

Além disso, buscou-se obter evidências de validade para o instrumento desenvolvido por meio de procedimentos de análise de componentes principais e de correlações com desempenho acadêmico e autorregulação da aprendizagem dos universitários. A expectativa era observar correlações negativas entre essas duas últimas variáveis e a percepção de consequências negativas da procrastinação em função de que indivíduos com melhor desempenho e com maior autorregulação da aprendizagem (ou seja, que conseguem administrar melhor seus esforços para aprender) devem procrastinar menos. Por fim, procurou-se verificar se a percepção dos respondentes sobre as consequências negativas da procrastinação acadêmica seria afetada pela própria atividade de responder ao instrumento.

\section{Método}

\section{Elaboração da versão preliminar do instrumento}

$O$ instrumento em construção foi denominado de Questionário de Procrastinação Acadêmica Consequências Negativas (QPROAC-CN). Alguns de seus itens foram inspirados em instrumentos tais como Tuckman Procrastination Scale (Tuckman, 1991), Procrastination Assessment Scale for Students (Solomon \& Rothblum, 1984), Pure Procrastination Scale (Steel, 2010),
Escala de Procrastinação Ativa (Gouveia et al., 2014) e Escala de Procrastinação Acadêmica (Sampaio, 2011).

A versão preliminar do QPROAC-CN possuía 80 itens distribuídos em 4 escalas com numeração independente:

- $\quad$ tarefas acadêmicas procrastinadas (5 itens);

- impacto geral da procrastinação acadêmica (10 itens);

- consequências negativas da procrastinação acadêmica (56 itens, englobando as dimensões de consequências acadêmicas, físicas e psicológicas); e

- motivação para mudança de comportamento (9 itens, englobando motivações para mudar e para buscar ajuda).

$\mathrm{Na}$ escala sobre as consequências negativas, 21 itens eram relativos às consequências acadêmicas, 18 itens às físicas e 17 itens às psicológicas. Todos os itens foram submetidos à análise de nove juízes que fizeram sugestões de ajustes e avaliaram a pertinência dos mesmos como indicadores do conteúdo do instrumento. Após a exclusão de itens em função das sugestões dos juízes, a versão final do instrumento ficou com 69 itens, divididos em seis escalas independentes. O QPROAC-CN foi aplicado na população somente após a realização dos ajustes sugeridos pelos juízes que excluíram os itens em função de aspectos semânticos e de repetição de ideias.

\section{Participantes}

Participaram desse estudo 534 estudantes de cursos de nível superior, recrutados via internet. Todos os participantes eram maiores de 18 anos, com média de idade de 24,92 anos $(D P=7,05)$, sendo 373 mulheres $(69,9 \%)$. Os cursos mais representados na amostra foram Administração (24,5\%), Psicologia $(19,0 \%)$ e Engenharias (8,9\%). A distribuição conforme a etapa dos cursos foi variada: $1^{\circ} / 2^{\circ}$ semestres $(13,1 \%), 3^{\circ} / 4^{\circ}(15,7 \%)$, $5 \% 6^{\circ}(23,6 \%), 7^{\circ} / 8^{\circ}(23,2 \%)$ e $9^{\circ}$ a $12^{\circ}(24,4 \%)$. Cerca de $67,4 \%$ dos participantes estudavam em uma instituição pública e o restante em instituições privadas de ensino superior. Do total, $60,5 \%$ estavam fazendo um curso superior pela primeira vez e $42,1 \%$ exerciam trabalho remunerado regular (exceto bolsas e estágios). A amostra utilizada $(n=534)$ foi composta pelos participantes que acessaram a pesquisa na internet e completaram o instrumento. O total de acessos registrado foi 822, o que caracteriza uma taxa de abandono de $35 \%$.

\section{Instrumentos}

\section{Questionário de Caracterização dos Participantes}

Foi utilizado um questionário com 20 perguntas para obtenção de informações demográficas e para caracterização da amostra. Neste questionário, foi avaliado o desempenho acadêmico autorrelatado, através da seguinte questão: "Em média, seu desempenho no curso / suas 
notas ficam em qual faixa percentual de aproveitamento? (assinale apenas uma): inferior a $50 \%$, de 50 a $59 \%$, de 60 a $69 \%$, de 70 a $79 \%$, de 80 a $89 \%$ e de 90 a $100 \% ”$.

\section{Questionário de Procrastinação}

Acadêmica - Consequências Negativas

Trata-se do instrumento construído e testado neste estudo. A escala Tarefas Acadêmicas Procrastinadas conta com cinco itens: "Procrastino fazer leituras acadêmicas", "Procrastino fazer exercícios acadêmicos", "Procrastino revisar e estudar conteúdos das disciplinas que estou cursando", "Procrastino estudar para provas da faculdade que possuem data marcada" e "Procrastino fazer trabalhos acadêmicos que tem prazo de entrega definido". Cada item é respondido em uma escala de cinco pontos

1. Nunca ou quase nunca;

2. Poucas vezes;

3. Algumas vezes;

4. Muitas vezes;

5. Sempre ou quase sempre; inclui ainda uma opção "não se aplica").

As demais escalas, Impacto Geral da Procrastinação Acadêmica, Consequências Negativas da Procrastinação Acadêmicae Motivaçãopara Mudançade Comportamento também são respondidas em uma escala de cinco pontos, mas com âncoras descritivas apenas nos extremos (1 A frase é totalmente falsa a meu respeito; 5 - A frase é totalmente verdadeira a meu respeito). Os itens destas escalas são descritos na seção de resultados.

\section{Inventário de Processos de}

\section{Autorregulação da Aprendizagem - Universidade}

O Inventário de Processos de Autorregulação da Aprendizagem - Universidade (IPAA) é uma escala unidimensional de autorrelato com oito itens que avalia processos da aprendizagem autorregulada de estudantes universitários, adaptada ao contexto brasileiro (Sampaio, 2011). O $\alpha$ de Cronbach é de 0,75 (Sampaio, 2011).

\section{Avaliação da percepção dos impactos da procrastinação após resposta ao Questionário de Procrastinação Acadêmica - Consequências Negativas}

Após a aplicação de todos os instrumentos, foi incluída uma questão final sobre a percepção dos participantes acerca dos impactos da procrastinação acadêmica antes e depois de participar da pesquisa, a saber: "A procrastinação acadêmica tem mais impactos na minha vida do que eu imaginava"; "A procrastinação acadêmica não me traz tantos impactos como eu imaginava"; e "Eu já tinha uma boa noção da quantidade de impactos que a procrastinação acadêmica tem na minha vida”.

\section{Procedimento}

Este projeto foi aprovado pelo Comitê de Ética em Pesquisa do Instituto de Psicologia da Universidade
Federal do Rio Grande do Sul (Parecer 89.727), e seguiu os padrões éticos de pesquisa com seres humanos (Resolução CNS no 466). Os instrumentos foram disponibilizados na internet por meio do site Survey Monkey (www.surveymonkey.com). Os potenciais participantes foram contatados por meio de convite eletrônico enviado à rede de contatos dos pesquisadores e para coordenações de cursos superiores, as quais foram solicitadas a repassar o convite da pesquisa aos alunos. Além disso, solicitou-se que cada participante repassasse o convite para outros universitários. A opção pela coleta online e o procedimento de solicitar o repasse do convite foram feitos por conveniência, para facilitar a obtenção de uma amostra grande.

Antes de ter acesso aos instrumentos, uma página inicial informava objetivos da pesquisa e exigia a concordância com um Termo de Consentimento Livre e Esclarecido (TCLE) para o prosseguimento da pesquisa. O universitário não tinha tempo limite para responder aos instrumentos e, caso ele desejasse um feedback do resultado, bastava deixar seu contato em um campo disponível para isso no final da pesquisa ou enviar um e-mail ao pesquisador responsável (esse e-mail constava no TCLE).

\section{Análise de dados}

O principal recurso de análise utilizado foi a análise de componentes principais (ACP), cujo objetivo é reduzir um determinado número de itens a um menor número de variáveis (Damásio, 2012). Essa análise foi feita para verificar, de modo exploratório, a estrutura subjacente às escalas do QPROAC-CN. O número de componentes a reter em cada escala foi determinado por uma combinação de critérios, como eigenvalue maior do que 1 , o método de análise paralela (Horn, 1965) e a interpretabilidade das dimensões. Para verificar a adequação da amostra para uso da ACP, utilizou-se o critério de Kaiser-Meyer-Olkin (KMO). Para estimar a confiabilidade dessa escala, escolheu-se o coeficiente alpha de Cronbach. Correlações de Pearson foram empregadas para verificar a relação entre as consequências negativas da procrastinação e as variáveis relativas ao processo de autorregulação da aprendizagem e ao desempenho acadêmico autorrelatado. Em função de o QPROAC-CN ser composto por quatro escalas independentes, optou-se por realizar análises independentes de cada uma dessas escalas e de suas subescalas que também podem ser usadas isoladamente.

\section{Resultados}

A Escala de Tarefas Acadêmicas Procrastinadas foi analisada levando em consideração cada um de seus cinco itens separadamente. Isso foi feito porque, embora seja possível calcular uma média dos itens e tomar a média como uma indicação da frequência geral de procrastinação acadêmica, nem todas as tarefas listadas podem ser aplicadas com igual peso à realidade de todos os estudantes, o que pode tornar o escore total médio 
uma estimativa enviesada da magnitude da procrastinação. Assim, optou-se por apresentar os dados descritivos de cada item individualmente: "procrastino fazer leituras acadêmicas" ( $M=4,30 ; D P=1,15)$; "procrastino fazer exercícios acadêmicos" $(M=4,02 ; D P=1,22)$; "procrastino revisar e estudar conteúdos das disciplinas que estou cursando" $(M=4,30 ; D P=1,22)$; "procrastino estudar para provas da faculdade que possuem data marcada" $(M=3,98 ; D P=1,40)$; e "procrastino fazer trabalhos acadêmicos que tem prazo de entrega definido" $(M=3,93$; $D P=1,36)$. Considerando a chave de respostas utilizada, verifica-se que, em média, para todos os tipos de tarefa, o escore esteve próximo de 4 , que significa procrastinar "muitas vezes" a tarefa descrita.

A análise inicial da Escala de Impacto Geral (10 itens, 534 casos com respostas completas) indicou que os dados eram adequados para a ACP $(\mathrm{KMO}=0,86$; teste de Bartlett com $p<0,001)$. Na análise inicial, foram identificados dois componentes com eigenvalues maiores do que 1 , e a análise paralela também sugeriu a extração de dois componentes. No entanto, apenas os itens 1 e 9 apresentaram cargas mais elevadas no segundo componente, o que levou à eliminação dos mesmos.

Uma nova ACP com os oito itens restantes mostrou-se adequada $(\mathrm{KMO}=0,87$; teste de Bartlett com $p<0,001)$, sendo identificados novamente dois componentes com eigenvalue maiores do que 1 . No entanto, a análise paralela sugeriu a existência de apenas um componente mais relevante. Dada a ambiguidade deste resultado, procurou-se interpretar qual seria o possível sentido dos dois componentes identificados, sendo que não se obteve uma interpretação clara para os mesmos. Por isso, optou-se pela solução unidimensional para esta escala, com variância explicada de 53,4\%. O $\alpha$ de Cronbach observado para esta escala foi de 0,84 . A Tabela 1 apresenta os itens e as cargas da Escala de Impacto Geral da
Procrastinação Acadêmica $(M=3,10 ; D P=0,94$; valor mínimo $=1$; valor máximo $=5$ ) .

Em relação à Escala de Consequências Acadêmicas (21 itens, 491 casos com respostas completas), também foi constatada adequação da amostra para uso da ACP $(\mathrm{KMO}=0,94$; teste de Bartlett $\operatorname{com} p<0,001)$. A primeira solução obtida indicou três componentes principais com eigenvalues maiores do que 1, enquanto a análise paralela indicou dois componentes mais relevantes. Os resultados mostraram ainda que os itens 11, 16 e 21 possuíam carga cruzada (ou seja, superior a 0,30 em mais de um componente) de magnitude próxima (com cargas variando entre 0,30 e 0,47 ), que foram então excluídos.

Em seguida, executou-se outra ACP, que revelou novamente três componentes principais com eigenvalues maiores do que $1(\mathrm{KMO}=0,92$; teste de Bartlett com $p<0,001)$. Porém a análise paralela sugeriu a extração de apenas dois componentes. Após uma análise semântica das soluções de três e dois componentes, optou-se pela solução de dois componentes (o primeiro explicando $50,8 \%$ da variância total e o segundo $12 \%$ ). O primeiro componente, com sete itens, foi denominado de "resultados acadêmicos", enquanto o segundo, formado pelos 11 itens restantes, que avaliam aspectos qualitativos envolvidos na realização das tarefas acadêmicas, foi chamado de "gestão das tarefas acadêmicas". O item 12, que apresentou carga cruzada, foi mantido por ter um peso mais consistente no segundo componente (superior a 0,40 ), além de ter conteúdo coerente com os demais itens. $\mathrm{O} \alpha$ de Cronbach observado para as escalas derivadas destes componentes foi de 0,88 para "resultados acadêmicos" $(M=1,80 ; D P=0,86$; valor mínimo $=1$; valor máximo $=5)$ e de 0,91 para "gestão das tarefas acadêmicas" $(M=2,91 ; D P=0,92$; valor mínimo $=1$; valor máximo=5). A Tabela 2 apresenta os itens e as cargas da Escala de Consequências Acadêmicas.

Tabela 1

Cargas Padronizadas na Análise de Componentes Principais dos Itens da Escala de Impacto Geral

\begin{tabular}{|c|c|}
\hline Item & Carga \\
\hline 2. Percebo que minha vida é bastante afetada por eu procrastinar minhas tarefas acadêmicas. & 0,80 \\
\hline 3. Procrastinar minhas tarefas acadêmicas gera mais consequências negativas do que positivas para mim. & 0,65 \\
\hline $\begin{array}{l}\text { 4. Percebo as reais consequências de procrastinar somente depois de ter procrastinado minhas } \\
\text { tarefas acadêmicas. }\end{array}$ & 0,59 \\
\hline $\begin{array}{l}\text { 5. As consequências negativas de eu procrastinar minhas tarefas acadêmicas estão ficando mais } \\
\text { frequentes com o passar do tempo. }\end{array}$ & 0,77 \\
\hline 6. Eu sinto uma sensação ruim por saber que procrastino minhas tarefas acadêmicas. & 0,78 \\
\hline 7. Procrastinar a realização de minhas tarefas acadêmicas diminui minha qualidade de vida. & 0,78 \\
\hline $\begin{array}{l}\text { 8. Eu continuo procrastinando minhas tarefas acadêmicas mesmo quando tenho muitos prejuízos em } \\
\text { função disso. }\end{array}$ & 0,76 \\
\hline $\begin{array}{l}\text { 10. As consequências negativas da procrastinação acadêmica na minha vida tem sido mais graves do } \\
\text { que eu costumo admitir. }\end{array}$ & 0,70 \\
\hline
\end{tabular}

Nota: apenas são exibidas as cargas superiores a 0,30. 
$\mathrm{Na}$ Escala de Consequências Físicas (18 itens, 534 casos com respostas completas), constatou-se que houve adequação da amostra para uso da análise de componentes principais $(\mathrm{KMO}=0,90$; teste de Bartlett com $p<0,001)$. Os três primeiros componentes principais apresentaram eigenvalues maiores do que 1 ; no entanto, a análise paralela indicou dois componentes para extração. Uma interpretação inicial das soluções com três e dois componentes sugeriu a retenção de apenas dois componentes. Além disso, os itens 3 e 17 apresentaram cargas cruzadas na solução de duas dimensões, tendo-se optado por retirá-los do conjunto. A análises foram então refeitas com 16 itens $(\mathrm{KMO}=0,89$; teste de Bartlett $\operatorname{com} p<0,001)$. Os itens 9, 10,11 e 18 tiveram cargas mais elevadas no primeiro componente, enquanto os demais itens foram carregados mais fortemente no segundo componente. Dois itens ainda apresentaram cargas maiores do que 0,30 nos dois componentes; por isso, considerou-se como ponto de corte carga de 0,40 para definir em qual dimensão esses itens seriam considerados. Os dois componentes explicaram $62,8 \%$ da variação total (48,1 e 14,7\%, respectivamente).

A análise do conteúdo semântico dos itens demonstrou que os quatro itens que formaram o primeiro componente estão vinculados ao uso de substâncias psicoativas (bebidas alcoólicas, fumo, uso de drogas ilícitas e medicamentos não-prescritos). Logo, esse componente foi denominado "uso de substâncias". Estas consequências, como pode ser observado pelas médias na Tabela 3, seriam menos frequentes como decorrência da procrastinação acadêmica. Já o segundo componente foi denominado "redução do autocuidado", e agrupou itens relacionados ao tempo dedicado ao descanso e à atividade física, à qualidade da alimentação, aos cuidados pessoais e à saúde em geral. $\mathrm{O} \alpha$ de Cronbach da subescala "uso de substância" foi $0,72(M=1,32 ; D P=0,64$; valor míni$m o=1$; valor máximo $=5$ ), e da subescala "redução do autocuidado" foi $0,91(M=2,56$; $D P=0,94$; valor mínimo $=1$; valor máximo $=5)$. Na Tabela 3 , são apresentados os itens e cargas da Escala de Consequências Físicas.

$\mathrm{Na}$ Escala de Consequências Psicológicas (17 itens, 534 casos com respostas completas), constatou-se que a amostra atendia às exigências para uso da análise de componentes principais $(\mathrm{KMO}=0,96$; teste de Bartlett com $p<0,001)$. A primeira solução obtida indicou dois componentes principais com eigenvalues maiores do que 1 , enquanto a análise paralela indicou apenas um componente.

Tabela 2

Cargas Padronizadas na Análise de Componentes Principais dos Itens da Escala de Consequências Acadêmicas

Item (por componente)

Componente

Procrastinar minhas tarefas acadêmicas faz com que...

1. ... eu deixe de estudar o essencial de cada disciplina.

0,56

2. ... eu estude somente o mínimo necessário para as provas.

0,66

3. ... eu não realize as leituras necessárias a tempo.

0,74

4. ... eu entregue meus trabalhos fora do prazo determinado.

0,63

5. ... eu não dê conta dos exercícios que são solicitados pelo professor.

0,59

6. ... eu tenha um rendimento ruim durante a primeira metade do semestre.

0,66

7.... eu tenha notas abaixo da média dos meus colegas.

0,76

8. ... eu desista de algumas disciplinas durante seu andamento.

0,90

9. ... eu reprove em disciplinas.

0,98

10. ... eu atrase o andamento do meu curso por não dar conta das disciplinas.

0,89

12. ... eu obtenha um resultado de qualidade inferior a que poderia obter caso eu não procrastinasse.

0,72

13. ... eu não consiga dar o meu melhor durante a realização dessas tarefas.

0,79

14.... eu tenha pressa no momento de realizar a tarefa.

0,88

15. ... eu tenha mais dificuldade para aprender o conteúdo das disciplinas.

0,32

0,49

17.... eu tenha constantemente tarefas acadêmicas acumuladas.

0,82

18. ... eu dedique muito tempo para dar conta de uma tarefa nos últimos instantes antes de se esgotar o prazo que devo cumprir (na véspera).

19. ... eu faça a tarefa de um jeito menos cuidadoso.

0,76

20. ... eu falte aula de uma disciplina para dar conta de outras tarefas acadêmicas.

Nota: apenas são exibidas as cargas superiores a 0,30. 
Os itens 6, 12 e 16 foram excluídos, pois apresentaram carga cruzada de magnitude próxima (cargas variando entre 0,34 e 0,52). Excluindo-se esses três itens, a amostra manteve-se adequada para uso da $\mathrm{ACP}(\mathrm{KMO}=0,95$; teste de Bartlett com $p<0,001$ ).

Logo após, executou-se outra ACP que, novamente, extraiu dois componentes principais com eigenvalues maiores do que 1 . No entanto, a análise paralela continuou sugerindo apenas um fator. Após uma análise semântica das duas últimas soluções, optou-se pela solução de dois componentes - o primeiro explicando $62,0 \%$ da variância total e o segundo $8,1 \%$. Os itens de número 1 a 9 , exceto o item 6 , formaram o primeiro componente, denominado "esgotamento psicológico". O segundo componente foi formado pelos outros cinco itens, e trata do quanto o universitário fica com sentimentos ruins a respeito de si mesmo, tais como irritação e vergonha em função de não dar conta da realização de suas tarefas acadêmicas. Esse componente foi denominado como "desapreço pessoal". $\mathrm{O} \alpha$ de Cronbach obtido para as escalas derivadas destes componentes foi de 0,92 para "esgotamento psicológico" $(M=3,03 ; D P=1,06$; valor mínimo $=1$; valor máximo=5) e de 0,94 para "desapreço pessoal" $(M=3,04 ; D P=1,27$; valor mínimo=1; valor máximo=5), indicando boa consistência interna. A Tabela 4, apresenta os itens e as cargas da Escala de
Consequências Psicológicas.Na escala sobre "mudança de comportamento" (9 itens, 522 casos com respostas completas), verificou-se que a amostra era adequada para uso da análise de componentes principais $(\mathrm{KMO}=0,84$; teste de Bartlett com $p<0,001)$. Na primeira análise, foram identificados dois componentes com eigenvalues maiores do que 1, e a análise paralela também sugeriu a extração de dois componentes. O item 3 foi eliminado por apresentar baixa carga e ambiguidade no que se refere à interpretação dos resultados.

A partir disso, executou-se outra ACP que extraiu dois componentes principais com eigenvalues maiores do que $1(\mathrm{KMO}=0,86$; teste de Bartlett $\operatorname{com} p<0,001)$. A análise paralela também sugeriu a existência de dois componentes principais. O primeiro deles é formado pelos itens 1, 2, 4 e 5, e foi denominado "necessidade de mudança". Este componente está relacionado à percepção de que há necessidade e/ou intenção de se reduzir a frequência com que as tarefas acadêmicas são procrastinadas. O segundo componente é formado pelos itens $6,7,8$ e 9 e trata do interesse do universitário em receber ajuda para procrastinar menos, denominado "interesse em ser ajudado". Esses dois componentes explicaram 82,2\% da variância total (o primeiro 64,4 e o segundo $17,8 \%)$. O $\alpha$ de Cronbach para a escala de "necessidade de mudança" foi de 0,87

Tabela 3

Cargas Padronizadas na Análise de Componentes Principais dos Itens da Escala de Consequências Físicas

\begin{tabular}{|c|c|c|}
\hline \multirow{2}{*}{ Item } & \multicolumn{2}{|c|}{ Componente } \\
\hline & I & II \\
\hline \multicolumn{3}{|l|}{ Procrastinar minhas tarefas acadêmicas faz com que... } \\
\hline 1.... eu tenha problemas na minha saúde física. & & 0,66 \\
\hline 2. ... eu engorde (ganhe peso corporal). & & 0,57 \\
\hline 4. ... eu tenha mais chances de ficar doente em função do meu desgaste físico. & & 0,73 \\
\hline 5. ... eu não realize regularmente meus exames de acompanhamento médico. & & 0,62 \\
\hline 6. ... eu não durma todo o tempo que eu precisaria dormir. & & 0,91 \\
\hline 7. ... eu tenha prejuízo na qualidade do meu sono. & & 0,93 \\
\hline 8. ... eu reduza o tempo que eu teria para descansar durante o dia (exceto dormir). & & 0,91 \\
\hline 9. ... eu fume mais do que costumo fumar. & 0,87 & \\
\hline 10. ... eu consuma bebidas alcoólicas além do que costumo beber. & 0,88 & \\
\hline 11. ... eu use drogas ilícitas (inalantes, maconha, crack, cocaína, etc). & 0,95 & \\
\hline 12. ... eu utilize em excesso alimentos estimulantes (café, chimarrão, chá preto, chocolate, etc). & & 0,60 \\
\hline 13. ... eu piore a qualidade da minha alimentação. & & 0,78 \\
\hline 14. ... minha vida sexual seja prejudicada. & & 0,55 \\
\hline 15. ... eu não consiga manter a prática de uma atividade física como eu gostaria. & & 0,78 \\
\hline $\begin{array}{l}\text { 16. ... eu não realize adequadamente minhas atividades de higiene (como escovar os } \\
\text { dentes e tomar banho). }\end{array}$ & 0,39 & 0,44 \\
\hline 18. ... eu tome medicamentos que não me foram prescritos. & 0,57 & 0,38 \\
\hline
\end{tabular}

Nota: apenas são exibidas as cargas superiores a 0,30. 
$(M=3,75 ; D P=1,09$; valor mínimo=1; valor máxi$\mathrm{mo}=5)$, e para a de "interesse em ser ajudado" foi de $0,92(M=2,72 ; D P=1,43$; valor mínimo=1; valor máximo $=5)$. A Tabela 5 apresenta os itens e as cargas da Escala de Mudança de Comportamento.
Evidências de validade complementares foram obtidas correlacionando-se o desempenho acadêmico autorrelatado e a autorregulação da aprendizagem com as escalas de consequências acadêmicas, psicológicas e físicas (subescalas de uma mesma escala foram combinadas para

Tabela 4

Cargas Padronizadas na Análise de Componentes Principais dos Itens da Escala de Consequências Psicológicas

Item (por componente principal)

Procrastinar minhas tarefas acadêmicas faz com que...

1. ... eu me sinta emocionalmente esgotado(a). $\quad 0,79$

$\begin{array}{lr}\text { 2. ... eu fique agitado(a). } & 0,89\end{array}$

$\begin{array}{lr}\text { 3. ... eu fique menos concentrado(a) e atento(a). } & 0,90\end{array}$

$\begin{array}{lr}\text { 4. ... eu fique mais ansioso(a) em situações de avaliação. } & 0,76\end{array}$

$\begin{array}{ll}5 . . . . \text { eu fique sem a energia necessária para realizar minhas tarefas acadêmicas. } & 0,79\end{array}$

7. ... eu perca meu interesse pelo curso em que estou matriculado(a). $\quad 0,62$

$\begin{array}{ll}\text { 8. ... eu fique nervoso(a) com tarefas simples que preciso realizar. } & 0,82\end{array}$

$\begin{array}{lc}\text { 9. ... eu me sinta sobrecarregado(a) por ter acumulado muitas tarefas. } & 0,67\end{array}$

10. ... eu sinta vergonha de mim mesmo(a) por procrastinar. 0,93

11. ... eu me irrite comigo por agir assim. 0,81

13. ... eu fique preocupado(a) com o que as pessoas pensam do meu comportamento. 0,84

14. ... eu sinta culpa por fazer isso com tarefas importantes para mim. 0,92

15. ... eu fique decepcionado(a) comigo. 0,93

17. ... eu fique frustrado(a) por não conseguir atingir outros objetivos de vida que 0,61 dependem da realização de minhas tarefas acadêmicas.

Nota: apenas são exibidas as cargas superiores a 0,30.

Tabela 5

Cargas Padronizadas na Análise de Componentes Principais dos Itens da Escala de Mudança de Comportamento

\begin{tabular}{|c|c|c|}
\hline \multirow{2}{*}{ Item } & \multicolumn{2}{|c|}{ Componente } \\
\hline & I & II \\
\hline $\begin{array}{l}\text { 1. Percebo que reduzir a frequência com que eu procrastino minhas tarefas acadêmicas } \\
\text { seria algo bom para mim. }\end{array}$ & 0,86 & \\
\hline $\begin{array}{l}\text { 2. Eu deveria reduzir urgentemente a frequência com que procrastino minhas tarefas } \\
\text { acadêmicas. }\end{array}$ & 0,75 & \\
\hline $\begin{array}{l}\text { 4. Tenho pensado no que posso fazer para diminuir o número de vezes que eu procrastino } \\
\text { minhas tarefas acadêmicas. }\end{array}$ & 0,84 & \\
\hline $\begin{array}{l}\text { 5. Estou disposto a me esforçar para reduzir a frequência com que procrastino minhas } \\
\text { tarefas acadêmicas. }\end{array}$ & 0,89 & \\
\hline $\begin{array}{l}\text { 6. Gostaria de ter ajuda de alguma pessoa para superar a tendência de procrastinar } \\
\text { minhas tarefas acadêmicas. }\end{array}$ & & 0,78 \\
\hline $\begin{array}{l}\text { 7. Tenho interesse de ser acompanhado por algum profissional para que eu procrastine } \\
\text { menos minhas tarefas acadêmicas. }\end{array}$ & & 0,95 \\
\hline $\begin{array}{l}\text { 8. Tenho vontade de fazer um tratamento psicológico individual para diminuir meu } \\
\text { sofrimento relacionado à procrastinação acadêmica. }\end{array}$ & & 0,94 \\
\hline $\begin{array}{l}\text { 9. Participaria de uma intervenção psicológica em grupo para diminuir a procrastinação de } \\
\text { minhas tarefas acadêmicas. }\end{array}$ & & 0,86 \\
\hline
\end{tabular}

Nota: apenas são exibidas as cargas superiores a 0,30. 
estas análises). O desempenho acadêmico autorrelatado apresentou correlação negativa e significativa com a escala de consequências acadêmicas $(r=-0,52 ; p<0,001)$, psicológicas $(r=-0,16 ; p<0,001)$ e físicas $(r=-0,11 ; p=0,010)$. Já a autorregulação da aprendizagem correlacionou-se negativamente e com significância estatística com o escore de consequências acadêmicas $(r=-0,30 ; p<0,001)$, mas não com as consequências psicológicas $(r=-0,08$; $p=0,084)$ e físicas $(r=-0,03 ; p=0,488)$.

Quando solicitados a comparar sua percepção sobre os efeitos da procrastinação acadêmica em suas vidas, antes e depois de participarem da pesquisa, cerca de $65 \%$ dos participantes afirmaram que já tinham uma boa noção da quantidade de impactos que a procrastinação acadêmica traz para suas vidas. Porém, 27,3\% dos universitários informaram que a procrastinação acadêmica tem mais impactos na vida deles do que eles imaginavam antes de responder ao questionário, e 7,5\% dos participantes concluíram que a procrastinação acadêmica não possui tantos impactos como eles imaginavam anteriormente.

\section{Discussão}

Este estudo buscou construir um instrumento para avaliar a percepção de estudantes universitários em relação às tarefas acadêmicas que eles procrastinam, o impacto geral da procrastinação acadêmica e suas consequências negativas em termos acadêmicos, físicos e psicológicos. Ademais, o instrumento busca avaliar a motivação para reduzir a procrastinação acadêmica e receber ajuda. As evidências de validade do instrumento foram obtidas através de procedimentos de análise de componentes principais e de correlações que, de modo geral, indicaram que a versão final do instrumento possui características psicométricas adequadas.

Há evidências de que, no contexto brasileiro, aproximadamente quatro em cada cinco universitários procrastinam suas tarefas acadêmicas (Geara, 2012) e mais da metade deles fazem isso ao menos uma vez por semana (Sampaio, 2011). Para evitar a procrastinação, os indivíduos podem se beneficiar de uma análise das possíveis consequências de seus atos, uma vez que isso pode ter impacto na probabilidade de ocorrência de um determinado comportamento (Rebetez et al., 2016). É possível que a procrastinação seja influenciada por uma dificuldade, por parte de quem procrastina, de antecipar as consequências que este comportamento pode ter no futuro, especialmente no que diz respeito à realização de objetivos (DeWitte \& Schouwenberg, 2002). Além disso, a dificuldade de reconhecer possíveis consequências negativas pode permanecer mesmo após a ocorrência da procrastinação, aumentando as chances de o indivíduo vir a procrastinar suas tarefas acadêmicas novamente.

Portanto, é fundamental que os indivíduos possam contar com ferramentas que facilitem essa reflexão.
Instrumentos como o QPROAC-CN podem colaborar com a conscientização a respeito da variedade e da frequência de ocorrência de consequências negativas da procrastinação acadêmica. Considerando-se que $27,3 \%$ dos participantes afirmaram que a procrastinação acadêmica tem mais impactos na vida deles do que eles imaginavam antes de responder ao questionário, pode-se entender que o instrumento cumpre com sua função de facilitar a reflexão crítica dos estudantes. Esse reconhecimento das consequências negativas da procrastinação acadêmica pode despertar no estudante a motivação para modificar seu comportamento por meio do desenvolvimento de sua capacidade de avaliar criticamente os riscos da procrastinação acadêmica antes de seguir procrastinando.

Outro objetivo deste estudo foi obter evidências de validade para o instrumento desenvolvido por meio de correlações do desempenho acadêmico dos universitários e da autorregulação da aprendizagem com as consequências negativas da procrastinação acadêmica. Para o desempenho acadêmico, observou-se, como esperado, relação negativa entre a percepção de consequências e desempenho, ou seja, quanto mais consequências negativas percebidas, pior o desempenho, sendo a relação mais forte observada para as consequências acadêmicas (para as consequências psicológicas e físicas a relação foi no sentido esperado, embora muito fraca).

Já a autorregulação da aprendizagem apresentou correlação significativa e negativa apenas com as consequências acadêmicas. A relação mais forte das consequências negativas acadêmicas tanto com o desempenho quanto com a autorregulação era esperada, uma vez que são variáveis mais diretamente ligadas ao âmbito das tarefas e resultados acadêmicos (Grunschel et al., 2013). A ausência de correlação da autorregulação com as consequências psicológicas e físicas necessita ser melhor explorada em estudos futuros. Afinal, é possível que pessoas com maior autorregulação sejam também mais preocupadas com o desempenho acadêmico e com isso desgastem-se mais física e psicologicamente para obter os resultados desejados, mesmo quando procrastinaram a realização da tarefa.

Dada a relevância da avaliação das consequências negativas da procrastinação acadêmica, sugere-se o uso do QPROAC-CN de duas maneiras:

1. para fins de pesquisa, na medida em que avalia a magnitude do fenômeno, permitindo relacioná-lo com medidas de outros construtos;

2. para fins clínicos ou educacionais, aproveitando-se mais o conteúdo de seus itens (aspecto qualitativo) em processos de avaliação da procrastinação acadêmica e de intervenção também.

Dessa maneira, o instrumento poderá servir como ferramenta para avanço na construção do conhecimento sobre o tema em diversos contextos.

Uma limitação deste estudo deve ser assinalada, que é a natureza da amostra que foi não probabilística e o fato de ter 
incluído, majoritariamente, estudantes de um único estado da federação. Logo, sugere-se que outras pesquisas sejam realizadas ampliando-se o perfil das amostras (diferentes cursos, regiões do país e características sociodemográficas) antes que tais achados sejam generalizados. Não obstante, ressalta-se que a quantidade de participantes foi adequada para realizar as análises estatísticas empregadas, o que faz pensar que os resultados obtidos possam ser replicados em outras amostras.

Outra limitação da pesquisa é o processo de autosseleção da amostra. É possível que aqueles que optaram por colaborar com o estudo sejam estudantes menos procrastinadores, já que tiveram o trabalho de responder ao instrumento. No entanto, o foco da pesquisa refere-se às tarefas acadêmicas e diversos estudantes comentaram, espontaneamente, ao final da pesquisa, que realizaram-na enquanto procrastinavam a realização de uma tarefa acadêmica. Isso implica dizer que a amostra dessa pesquisa pode ter sido composta por estudantes que procrastinam tanto que chegam a responder uma pesquisa sobre o tema enquanto deveriam estar realizando suas tarefas acadêmicas.

Para futuras pesquisas, sugere-se a avaliação de possíveis interações entre as consequências da procrastinação acadêmica e seus impactos sobre a motivação dos indivíduos para modificar seu comportamento. Possíveis distinções de tipos de estudantes que procrastinam, com base nas consequências mais comuns que eles experimentam, também podem ser úteis para a elaboração de programas de prevenção e de intervenção nesse âmbito. Desta for$\mathrm{ma}$, as iniciativas existentes que se propõem a intervir no contexto da procrastinação, tais como oficinas de gestão do tempo com universitários (Basso, Graf, Lima, Schmidt, \&
Bardagi, 2013; Furlan, 2013; Oliveira, Carlotto, Teixeira, \& Dias, 2016), podem ser aprimoradas continuamente.

Considerando-se os resultados apresentados, verifica-se que o QPROAC-CN possui evidências de validade de construto (obtidas por meio da análise de componentes principais) e de precisão (obtidas por meio da avaliação da consistência interna pelo alfa de Cronbach). Assim, fica justificado o seu emprego, no contexto brasileiro, para medição das consequências negativas da procrastinação acadêmica dos universitários.

Em sala de aula, o instrumento pode ser utilizado tanto para avaliação e registro do comportamento dos estudantes como para levantamento de possíveis demandas para posterior desenvolvimento de atividades que promovam a autorregulação da aprendizagem dos estudantes universitários. A partir do mapeamento de quais consequências negativas da procrastinação acadêmica estão mais presentes em um determinado grupo de estudantes, torna-se mais ágil o processo orientação dos mesmos e de elaboração de programas de prevenção relacionados ao tema. Nessas intervenções, o instrumento pode ser útil enquanto dispositivo de promoção da reflexão dos estudantes sobre esse tema, a partir da discussão coletiva ou da análise individual dos itens do instrumento.

\section{Agradecimentos}

Os autores agradecem à Fundação de Amparo à Pesquisa do Estado do Rio Grande do Sul pelo apoio à pesquisa na forma de bolsa de mestrado concedida à primeira autora.

\section{Referências}

Bandura, A. (1991). Self-regulation of motivation through anticipatory and self- reactive mechanisms. Em R. A. Dienstbier (Ed.), Perspectives on motivation: Nebraska symposium on motivation. (pp.69-164). Lincoln: University of Nebraska Press.

Basso, C., Graf, L. P., Lima, F. C., Schmidt, B., \& Bardagi, M. P. (2013). Organização de tempo e métodos de estudo: oficinas com estudantes universitários. Revista Brasileira de Orientação Profissional, 14(2), 277-288. Recuperado de http://pepsic.bvsalud.org/scielo. php?script $=$ sci_arttext\&pid $=$ S1679-33902013000200012

Couto, M. C. P. P. (2014). Como manejar o tempo na academia. Em S. H. Koller, M. C. P. P. Couto, \& J. V. Hohendorff (Orgs.), Manual de produção científica. (pp. 167-177). Porto Alegre, RS: Penso.

Damásio, B. F. (2012). Uso da análise fatorial exploratória em psicologia. Avaliação Psicológica, 11(2), 213-228. Recuperado de http://pepsic. bvsalud.org/scielo.php?script $=$ sci_arttext\&pid $=$ S1677-04712012000200007

Dewitte, S., \& Schouwenburg, H. C. (2002). Procrastination, temptations and incentives: The struggle between the present and the future in procrastination and the punctual. European Journal of Personality, 16(6), 469-489. doi: 10.1002/per.461

Ferrari, J., O'Callaghan, J., \& Newbegin, I. (2005). Prevalence of procrastination in the United States, United Kingdom, and Australia: Arousal and avoidance delays among adults. North American Journal of Psychology, 7(1), 1-6.

Furlan, L. A. (2013). Eficacia de una intervención para disminuir la ansiedad frente a los exámenes en estudiantes universitários argentinos. Revista Colombiana de Psicología, 22(1), 75-89. Recuperado de http://www.revistas.unal.edu.co/index.php/psicologia/article/ view/20784/43286

Geara, G. B. (2012). Procrastinação acadêmica em estudantes universitários: construção de um instrumento e evidências de validade. (Trabalho de conclusão de curso). Universidade Federal do Rio Grande do Sul, Porto Alegre. pp. 01-50.

Gouveia, V. V., Pessoa, V. S., Coutinho, M. L., Barros, I. C. S., \& Fonseca, A. A. (2014). Escala de procrastinação ativa: evidências de validade fatorial e consistência interna. Psico-USF, 19(2), 345-354. doi: 10.1590/1413-82712014019002008

Grunschel, C., Patrzek, J., \& Fries, S. (2013). Exploring reasons and consequences of academic procrastination: An interview study. European Journal of Psychology of Education, 28(3), 841-861. doi: 10.1007/s10212-012-0143-4 
Horn, J. L. (1965). A rationale and test for the number of factors in factor analysis. Psychometrika, 30(2), 179-185. doi: 10.1007/BF02289447

Hussain, I., \& Sultan, S. (2010). Analysis of procrastination among university students. Procedia - Social and Behavioral Sciences, 5, $1897-1904$. doi:10.1016/j.sbspro.2010.07.385

Klingsieck, K. (2013). Procrastination: When good things don't come to those who wait. European Psychologist, 18(1), 24-34. doi: 10.1027/1016-9040/a000138

Krause, K., \& Freund, A. M. (2014). Delay or procrastination - A comparison of self-report and behavioral measures of procrastination and their impact on affective well-being. Personality and Individual Differences, 67, 75-80. doi: 10.1016/j.paid. 2014.01.050

O'Brien, W. K. (2002). Applying the transtheoretical model to academic procrastionation. (Dissertação de mestrado). University of Houston.

Oliveira, C. T., Carlotto, R. C., Teixeira, M. A. P., \& Dias, A. C. G. (2016). Oficinas de gestão do tempo com estudantes universitários. Psicologia: Ciência e Profissão, 36(1), 224-233. doi: 10.1590/1982-3703001482014

Polydoro, S. A. J., Pelissoni, A. M. S., Carmo, M. C., Emilio, E. R. V., Dantas, M. A., \& Rosário, P. (2015). Promoção da autorregulação da aprendizagem na universidade: percepção do impacto de uma disciplina eletiva. Revista de Educação PUC-Campinas, 20(3), $201-213$. Recuperado de http://periodicos.puc-campinas.edu.br/seer/index.php/reveducacao/article/view/2877

Rebetez, M. M. L., Barsics, C., Rochat, L., D’Argembeau, A., \& Linden, M. V. (2016). Procrastination, consideration of future consequences, and episodic future thinking. Consciousness and Cognition, 42(1), 286-292. doi: 10.1016/j.concog.2016.04.003

Sampaio, R. K. N. (2011). Procrastinação acadêmica e a autorregulação da aprendizagem em estudantes universitários. (Dissertação de Mestrado). Universidade Estadual de Campinas, Campinas. pp. 1-134.

Schouwenburg, H. (1995). Academic procrastination: Theoretical notions, measurement, and research. Em J. Ferrari, J. Johnson, \& W. McCown. Procrastination and task avoidance: Theory, research and practice. (pp. 71-95). New York: Plenum Press.

Schouwenburg, H. (2004). Trait procrastination in academic settings: An overview of students who engage in task delays. Em H. Schouwenburg, C. Lay, T. Pylchyl, \& J. Ferrari, (Eds.), Counselling the procrastinator in academic settings. (pp. 3-18). Washington: American Psychological Association.

Solomon, L. J., \& Rothblum, E. (1984). Academic procrastination: Frequency and cognitive-behavioural correlates. Journal of Counselling Psychology, 31(4), 503- 509. doi: 10.1037/0022-0167.31.4.503

Steel, P. (2007). The nature of procrastination: A meta-analytic and theoretical review of quintessential self-regulatory failure. Psychological Bulletin, 133(1), 65-94. doi:10.1037/0033-2909.133.1.65

Steel, P. (2010). Arousal, avoidant and decisional procrastinators: Do they exist? Personality and Individual Differences, 48(8), 926-934. doi:10.1016/j.paid.2010.02.025

Steel, P., \& Klingsieck, K. B. (2016). Academic procrastination: Psychological antecedents revisited. Australian Psychologist, 51(1), 36-46. doi: 10.1111/ap.12173

Tuckman, B. W. (1991). The development and concurrent validity of the procrastination scale. Educational and Psychological Measurement, 51(2), 473-480. doi: 10.1177/0013164491512022

\section{Sobre os autores}

Gabriela Ballardin Geara é Psicóloga, mestre em Psicologia e doutoranda em Psicologia pela Universidade Federal do Rio Grande do Sul (UFRGS).

Marco Antônio Pereira Teixeira é Doutor em Psicologia e professor no Instituto de Psicologia da UFRGS. Coordena o Núcleo de Estudos e Intervenções em Carreira (NEIC), o Núcleo de Apoio ao Estudante (NAE-UFRGS) e o Serviço de Orientação Profissional (SOP-UFRGS). 